\title{
Inhibition of Gli1-mediated prostate cancer cell proliferation by inhibiting the mTOR/S6K1 signaling pathway
}

\author{
HONG YANG* ${ }^{*}$ LIBING HU*, ZHIMIN LIU, YANG QIN, RUIQIAN LI, GUOYING ZHANG, \\ BIN ZHAO, CHENGWEI BI, YONGHONG LEI and YU BAI
}

Department of Urology, The Third Affiliated Hospital of Kunming Medical University, Kunming, Yunnan 650118, P.R. China

Received January 10, 2016; Accepted June 2, 2017

DOI: $10.3892 / 01.2017 .7254$

\begin{abstract}
Ectopic activation of the canonical Hedgehog signaling pathway is involved in the development and progression of prostate cancer, which is one of the leading causes of cancer-associated mortality in males worldwide. However, the role of the non-canonical Hedgehog signaling pathway in prostate cancer remains generally unexplored. In the present study, it was identified that Gli (glioma-associated oncogene) 1 and Gli2 were highly expressed at the protein level in the androgen-independent prostate cancer cell lines PC3 and DU145, but not in the androgen-dependent cancer cell line LNCaP. Silencing of Glil using small interfering RNA markedly decreased PC3 cell viability and liquid colony formation in vitro. The Gli1/2-specific inhibitor GANT61 markedly decreased cell viability by inducing cell apoptosis in PC3 and DU145 cells. GANT61 also alleviated liquid colony formation efficiency in PC3 and DU145 cells, suggesting that the activity of Glil is required for prostate cancer cell survival. To explore further the upstream signaling pathway involved in the regulation of Gli1 expression, it was identified that tumor necrosis factor $\alpha$-triggered mammalian target of rapamycin $(\mathrm{mTOR}) / \mathrm{p} 70$ ribosomal protein S6 kinase 1 (S6K1) activation was required for Gli1 expression. Pharmacological and genetic inhibition of S6K1 activation markedly decreased Gli1 and its downstream target gene mRNA expression. In addition, the phosphoinositide 3-kinase/mTOR inhibitor BEZ235 markedly decreased in vitro PC3 cell proliferation. The results of the present study indicate that the non-canonical Hedgehog pathway (mTOR/S6K1/Gli1) contributes to the development and progression of prostate cancer and that Gli1 is a potential therapeutic target in the treatment of prostate cancer.
\end{abstract}

Correspondence to: Dr Yu Bai, Department of Urology, The Third Affiliated Hospital of Kunming Medical University, 519 Kunzhou Road, Kunming, Yunnan 650118, P.R. China

E-mail: baiyuff@126.com

${ }^{*}$ Contributed equally

Key words: mammalian target of rapamycin/S6 kinase 1, glioma-associated oncogene 1 , prostate cancer, cell viability, Hedgehog signaling pathway

\section{Introduction}

Prostate cancer is the second most common malignancy in males worldwide, characterized by its high mortality and poor prognosis (1). In spite of early detection techniques and multidisciplinary therapeutic approaches, the overall 5-year survival rate remains $<30 \%$ in China (2). Anti-androgen treatment remains the first-line therapy for patients with prostate cancer, among whom the majority will eventually develop highly metastatic androgen-independent prostate cancer (3). Prostate cancer is a complex and heterogeneous disease characterized by various molecular signatures in patients, which provides opportunities to explore targeted therapies.

Glioma-associated oncogene 1 (Gli1) is highly expressed in the prostate and serves various functions in prostate development $(4,5)$. Ectopic activation of the Hedgehog ( $\mathrm{HH})$ signaling pathway has been demonstrated to be involved in the initiation as well as the progression of prostate malignancy $(3,6)$. The HH signaling pathway was first identified in Drosophila as a central organizer for proper embryonic patterning and development. Three HH ligands have been identified in vertebrate organisms: Sonic Hedgehog (SHH), Indian Hedgehog and Desert Hedgehog, which are all able to initiate signaling by binding and inactivating the $\mathrm{HH}$ receptor Patched 1 (PTCH1) (5). In the canonical HH signaling pathway, inactivation of PTCH1 releases the seven-pass transmembrane protein Smoothened (SMO). SMO then transduces the signal to downstream effectors (Gli proteins) via blocking the inhibitory partner, suppressor of fused. Activated Gli proteins eventually translocate into the nucleus and trigger the transcription of downstream target genes $(5,7)$. In addition to classical $\mathrm{HH}$ signal transduction, the non-canonical $\mathrm{HH}$ pathway, in which Gli proteins are regulated by phosphoinositide 3-kinase (PI3K)/V-Akt murine thymoma viral oncogene (Akt), mitogen-activated protein kinase (MAPK)/extracellular-signal-regulated kinase (ERK), nuclear factor $\kappa \mathrm{B}(\mathrm{NF}-\kappa \mathrm{B})$ and/or transforming growth factor $\beta$ (TGF $\beta$ ) pathways as well as the key tumor suppressors tumor protein p53 (TP53) and phosphatase and tensin homolog (PTEN) in a ligand-independent manner, has been the focus of previous research (8-10). Although the canonical pathway has been well-investigated, how Gli proteins are regulated in a SMO-independent manner in prostate cancer remains largely unknown. 
The Akt/mammalian target of rapamycin (mTOR)/p70 ribosomal protein S6 kinase 1 (S6K1) signaling pathway is involved in numerous aspects of molecular and cellular biology, such as mRNA translation, ribosome biogenesis, cell proliferation, metabolism, immunosuppression, development, aging and malignancies (8). As a serine/threonine protein kinase, the activation of mTOR leads to the phosphorylation of S6K1 and eukaryotic translation initiation factor 4E-binding protein 1 (4E-BP1) (11). S6K1 is also a serine/threonine kinase and serves a role in target gene translation following its phosphorylation by mTOR. More importantly, tumor necrosis factor $\alpha(\mathrm{TNF} \alpha)$ is capable of activating the mTOR/S6K1 signaling pathway to promote angiogenesis, which mediates chronic inflammation-induced cancer, including breast and prostate cancer $(8,12)$.

In order to explore the potential therapeutic targets for prostate cancer, an initial siRNA screen was performed (data not shown) and it was identified that Gli1 and Gli2 are critical in prostate cancer survival rates. Combined with other published data, it was hypothesized that Gli1 and/or Gli2 contributes to prostate cancer cell proliferation. In the present study, it was demonstrated that androgen-independent prostate cancer cell lines are dependent on Gli1 expression for proliferation and that its activation by mTOR/S6K1 is required for this function.

\section{Materials and methods}

Cell culture. The human prostate cancer cell lines PC3 (CRL-1435 $\left.{ }^{\mathrm{TM}}\right)$, DU145 (HTB-81 ${ }^{\mathrm{TM}}$ ) and LNCaP (CRL-1740 ${ }^{\mathrm{TM}}$ ) were purchased from the American Type Culture Collection (Manassas, VA, USA). The prostate cancer cell lines were maintained in RPMI-1640 medium (Sigma-Aldrich; Merck KGaA, Darmstadt, Germany) supplemented with $10 \%$ fetal bovine serum (FBS; cat. no. 10082147 Thermo Fisher Scientific, Waltham, MA, USA) and cultured at $37^{\circ} \mathrm{C}$ in a humidified atmosphere containing $5 \% \mathrm{CO}_{2}$. All cell lines were confirmed to be Mycoplasma-free using an e-Myco kit (Boca Scientific Inc., Boca Raton, FL, USA). The inhibitors used were GANT61 (Tocris Bioscience, Bristol, UK), rapamycin (Sigma-Aldrich; Merck KGaA) and BEZ235 (Selleck Chemicals, Houston, TX, USA).

Reverse transfection in prostate cancer cells with small interfering RNA (siRNA) targeting Gli1 or S6K1. SiRNA screening was performed as follows. The genome wide siRNA library was purchased from Dharmacon (Lafayette, CO, USA). The library contained a mixture of 4 individual siRNA oligos for each gene. PC3 cells were cultured in RPMI-1640 medium supplemented with $10 \% \mathrm{FBS}$ at $37^{\circ} \mathrm{C}$ for three days to reach $60-70 \%$ confluency. A total of 10 pmol of each siRNA pool $(5 \mu \mathrm{l})$ was reverse transfected to $95 \mu \mathrm{l}$ serum-free media in empty 96-well assay plates. Firstly, $5 \mu \mathrm{l}$ siRNA was diluted in $25 \mu \mathrm{l}$ OptiMEM (cat. no. 31985-062; Thermo Fisher Scientific, Inc.) serum-free medium and incubated for $5 \mathrm{~min}$ at room temperature. Then, $0.13 \mu 1$ RNAiMax (cat. no. 13778075; Thermo Fisher Scientific, Inc.) was diluted in $10 \mu$ l OptiMEM medium. Secondly, siRNA was mixed with Lipofectamine ${ }^{\circledR}$ RNAiMax (cat. no. 13778075; Thermo Fisher Scientific, Inc.) transfection reagent, incubated for $15 \mathrm{~min}$ at room temperature and transferred to 96-well plates. Thirdly, PC3 cells were harvested by incubation with trypsin for $2-4 \mathrm{~min}$ at $37^{\circ} \mathrm{C}$ and centrifugation for $5 \mathrm{~min}$ at $100 \mathrm{x} \mathrm{g}$ and $4^{\circ} \mathrm{C}$. A total of $\sim 2,500$ cells in $60 \mu \mathrm{l}$ cell suspension were seeded in each well and cultured for $96 \mathrm{~h}$ at $37^{\circ} \mathrm{C}$. CellTiter-Glo Assay kit (Promega Corporation, Madison, WI, USA) was used to measure the cell viability. Cells were plated onto a 96-well plate at a density of 2,500 cells/well. After $96 \mathrm{~h}$ incubation at $37^{\circ} \mathrm{C}$, CellTiter-Glo reagent was added to the culture medium, and plates were agitated at room temperature for $10 \mathrm{~min}$. The luminescent signal was determined using a GloMax absorbance plate reader at a wavelength of $560 \mathrm{~nm}$. siUBB and siTMEM114 were used as positive and negative controls, respectively. Each screening was triplicated and repeated three times. For $\mathrm{Z}$ scores, -3 was a cut-off value. The siRNAs targeting human Gli1 or S6K1 were synthesized by Sigma-Aldrich; Merck KGaA (siGli1: NM_005269, siS6K1: NM_003161). A scrambled siRNA (SIC001, Sigma-Aldrich; Merck KGaA) was used as a negative control. Transient knockdown of Gli1 or S6K1 with these siRNAs in prostate cancer cells was carried out using Lipofectamine RNAiMax (Thermo Fisher Scientific, Inc.) together with negative or positive controls (GAPDH siRNA, NM_002046; Sigma; Merck KGaA), according to the manufacturer's protocol. The cells were then cultured for $72 \mathrm{~h}$ at $37^{\circ} \mathrm{C}$ in $5 \% \mathrm{CO}_{2}$. Silencing efficiency was determined using the reverse transcription-quantitative polymerase chain reaction (RT-qPCR) and western blotting.

$R T-q P C R$. Total RNA was extracted from cultured cells using the RNeasy Mini kit (Qiagen, Valencia, CA, USA). cDNA was generated with oligo-dT primers from $0.5 \mu \mathrm{g}$ RNA using an iScript cDNA synthesis kit (Bio-Rad Laboratories, Inc., Hercules, CA, USA) according to the manufacturer's protocol. TaqMan probes (Thermo Fisher Scientific, Inc.) of Gli1, Gli2, N-Myc, PTCH1 and CCND1 genes were used to quantitatively analyze mRNA transcript levels with the $18 \mathrm{~S}$ ribosomal RNA gene as an internal reference. PCR was performed using the ABI 7300 Real-Time PCR system (Applied Biosystems; Thermo Fisher Scientific, Inc.). The thermocycling conditions were $95^{\circ} \mathrm{C}$ for $3 \mathrm{~min}, 45$ cycles at $95^{\circ} \mathrm{C}$ for $15 \mathrm{sec}$ and $60^{\circ} \mathrm{C}$ for $45 \mathrm{sec}$. The primers were Gli1 (Hs00171790_m1), Gli2 (Hs01119974_m1), N-Myc (Hs00190768_m1), PTCH1 (Hs00181117_m1), CCND1 (Hs00765553_m1), and reference gene GAPDH (Hs002786624_g1). All primers were purchased from Thermo Fisher Scientific, Inc. The results were analyzed using SDS Software v1.4.1 (Thermo Fisher Scientific, Inc.). The comparative $\mathrm{C}_{\mathrm{q}}$ method was used to calculate relative mRNA expression levels (13).

Cell viability assays. Cell viability was examined using the CellTiter-Glo Assay kit (Promega Corporation), according to the manufacturer's protocol. Cells were plated onto a 96 -well plate at a density of 2,000 cells/well. At 24,48, 72 and 96 h, CellTiter-Glo reagent was added to the culture medium, and plates were agitated at room temperature for $10 \mathrm{~min}$. The half-maximal inhibitory concentration was the concentration of GANT61 required for $50 \%$ inhibition of the cell viability in the curve. The luminescent signal was determined using a GloMax absorbance plate reader at a wavelength of $560 \mathrm{~nm}$. Each individual experiment was performed at least three times independently.

Liquid colony formation assays. To evaluate anchoragedependent liquid colony formation efficiency, 500 cells were 
A

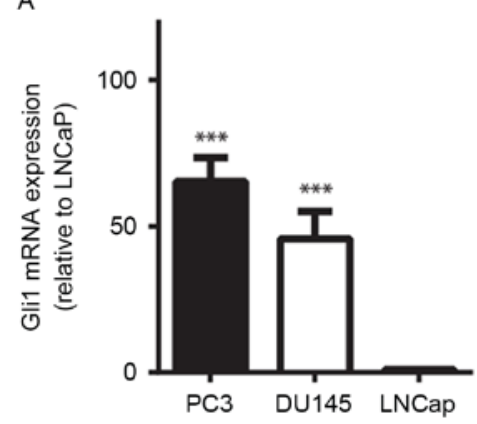

C

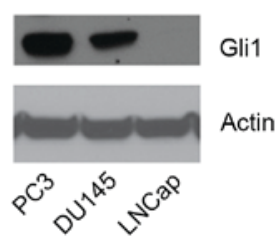

B

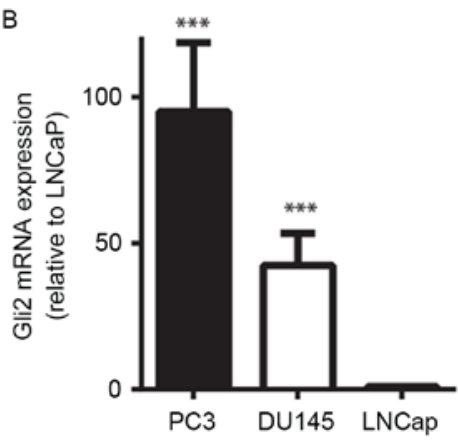

D

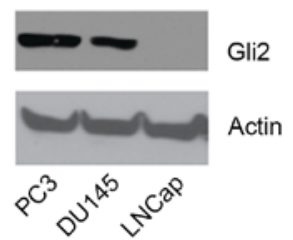

Figure 1. Gli1 and Gli2 are overexpressed in androgen-independent prostate cancer cell lines. (A) Gli1 and (B) Gli2 mRNA expression in prostate cancer cell lines PC3, DU145 and LNCaP was detected using the reverse transcription-quantitative polymerase chain reaction (fold expression compared with LNCaP). (C) Gli1 and (D) Gli2 protein expression was detected by immunoblotting lysates from PC3, DU145 and LNCaP cells. $\beta$-actin was used as a loading control. Results are presented as the mean \pm standard deviation. ${ }^{* * *} \mathrm{P}<0.001$ vs. control. Gli, glioma-associated oncogene.

suspended in $3 \mathrm{ml}$ RPMI-1640 medium with 10\% FBS and plated in a 6-well plate to grow for 2-3 weeks. The cells were fixed and stained with $1 \mathrm{ml} 0.05 \%$ crystal violet (Sigma-Aldrich; Merck KGaA) to count the colonies. Each individual experiment was performed three times independently.

Western blotting. Treated cells were lysed in Protein Extraction Reagent Type 4 (Sigma-Aldrich; Merck KGaA) with a PhosStop phosphatase inhibitor and cOmplete protease inhibitor (Roche Diagnostics, Indianapolis, IN, USA). The protein concentration of cell lysates was measured using the Bradford reagent (Bio-Rad Laboratories, Inc.). Equal amounts of total protein $(30 \mu \mathrm{g})$ were separated by SDS-PAGE on an 8-10\% gel, and then transferred onto nitrocellulose membranes (Bio-Rad Laboratories, Inc.). The membranes were blocked in 5\% milk followed by incubation with primary antibodies in Tris-buffered saline with $0.1 \%$ Tween-20 solution at $4^{\circ} \mathrm{C}$ overnight. The following day, the membranes were incubated with corresponding horseradish peroxidase-conjugated secondary antibodies: Goat anti-mouse immunoglobulin (Ig)G (cat. no. ab6789; Abcam, Cambridge, MA, USA; 1:3,000) and goat anti-rabbit IgG antibodies (cat. no. ab97051; Abcam; 1:3,000) for $1 \mathrm{~h}$ at room temperature. Membranes were exposed to LucentBlue X-ray film (Advansta, Menlo Park, CA, USA) at room temperature for between $30 \mathrm{sec}$ and $5 \mathrm{~min}$. Antibodies used for western blotting were as follows: Anti-Gli1 (cat. no. 2643), anti-poly(ADP-ribose) polymerase (PARP)/cleaved PARP (cat. no. 9546) and anti-phospho-S6K1 (Thr421/Ser424; cat. no. 9204; Cell Signaling Technology, Inc., Danvers, MA, USA); anti-S6K1 (cat. no. ab14708; Abcam); anti-Gli2 (cat. no. ABN506; EMD Millipore, Billerica, MA, USA); and anti- $\beta$-actin (cat. no. sc-4778; Santa Cruz Biotechnology, Dallas, TX, USA).

Statistical analysis. Student's t-test (two-tailed) was used to examine the significance of in vitro cell viability and quantitative real-time PCR data between different groups. $\mathrm{P}<0.05$ was considered to indicate a statistically significant difference.

\section{Results}

Overexpression of Glil and Gli2 in androgen-independent prostate cancer cell lines. Kim et al (4) demonstrated that the expression of certain $\mathrm{HH}$ signaling proteins was significantly associated with poor prognosis, including larger tumor size, increased level of prostate-specific antigen (PSA), higher Gleason score and poorer invasiveness. In the present study, it was examined whether Gli expression is associated with androgen dependency in cultured prostate cancer cells. RT-qPCR revealed that mRNA expression of Gli1 and Gli2 in the androgen-independent prostate cancer cell lines PC3 and DU145 was significantly increased compared with that in LNCaP cells, which is an androgen-sensitive cell line ( $\mathrm{P}<0.001$; Fig. 1A and B). Immunoblotting of the cell lysates using anti-Gli1 or -Gli2 antibody identified that Gli1 and Gli2 were overexpressed at the protein level in PC3 and DU145 cells compared with LNCaP cells, in which both Gli1 and Gli2 protein were barely detected (Fig. 1C and D). These results indicate that the $\mathrm{HH}$ signaling pathway may serve a critical role in androgen-independent prostate cancer cells.

Gli1 depletion decreases prostate cancer cell viability and liquid colony formation. To investigate whether endogenous Gli1 serves a role in prostate cancer cell proliferation, an siRNA targeting Gli1 (siGli1) was used to transiently knock down the expression of Gli1 in the PC 3 cell line. RT-qPCR and western blotting demonstrated that siGlil significantly decreased Gli1 mRNA and protein expression in PC3 cells $(\mathrm{P}<0.01$; Fig. $2 \mathrm{~A}$ and $\mathrm{B})$. In vitro liquid colony formation assays revealed that knocking down Gli1 resulted in a 
A

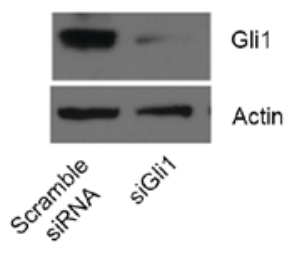

C

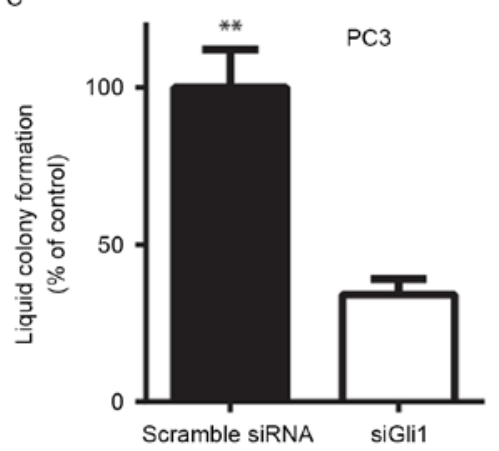

B

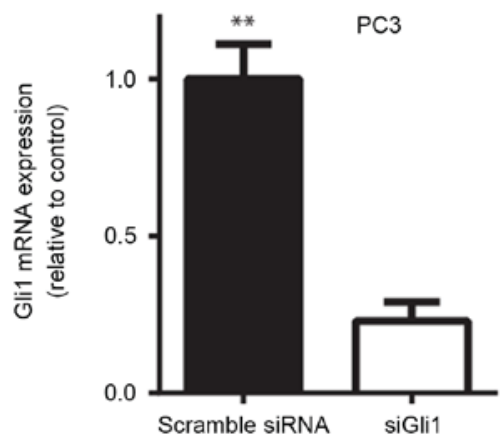

D

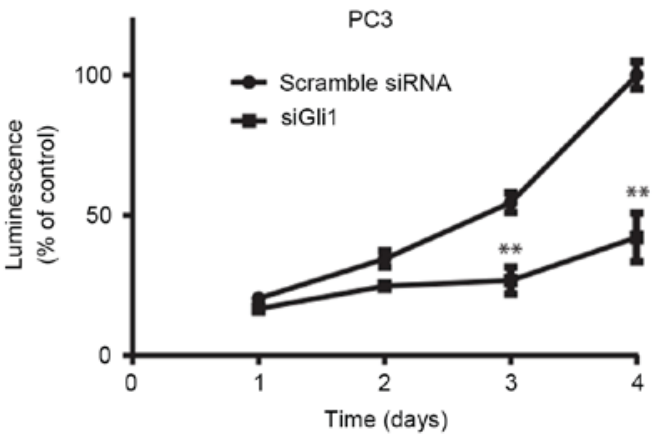

Figure 2. Knocking down of Glil leads to cell proliferation arrest and decreased cell clonogenicity in PC3 cells in vitro. (A) PC 3 cells were transiently transfected with siRNA targeting Gli1 or a scrambled siRNA. Depletion of Gli1 protein expression in PC 3 cells was examined by western blotting. $\beta$-actin was used as a loading control. (B) The reverse transcription-quantitative polymerase chain reaction revealed that siGli1 significantly decreased Gli1 mRNA levels in transfected PC3 cells. (C) The capability of PC3 cells to form colonies in liquid medium following depletion of Gli1 expression was significantly decreased. The number of colonies is expressed as a percentage of the control. (D) PC3 cells were seeded and cultured for 4 days, and cell viability was assessed using the CellTiter-Glo assay. The proliferation rate is presented as a percentage of the scramble control. ${ }^{* *} \mathrm{P}<0.01$ vs. control. Gli, glioma-associated oncogene 1 ; siRNA, small interfering RNA; siGli1, siRNA targeting Gli1.

significant decrease in anchorage-dependent colony formation efficiency in PC3 cells ( $\mathrm{P}<0.01$; Fig. 2C). Similarly, depletion of Gli1 expression led to a significant decrease in cell viability determined by measuring the ATP level in PC3 cells $(\mathrm{P}<0.01$; Fig. 2D). These results indicated that Gli1 is required for androgen-independent prostate cancer cell survival in vitro.

Gli-specific small molecule inhibitor GANT61 suppresses prostate cancer cell proliferation. Inhibiting Glil function may be a potential therapeutic strategy for the treatment of prostate cancer. A number of small-molecule inhibitors targeting Gli family proteins have been developed $(14,15)$. In the present study, it was examined whether the Gli1/2-specific inhibitor GANT61 was able to suppress prostate cancer cell viability. Cell viability assays revealed that GANT61 inhibited both PC3 and DU145 cell viability in vitro. The underlying molecular mechanisms by which GANT61 causes cell death are not fully understood, although a previous study demonstrated that GANT61 was able to inhibit the binding of Gli1/2 to the target gene promoter regions (16). PC3 cells exhibited slightly increased sensitivity to GANT61 compared with DU145 cells, and their half-maximal inhibitory concentration values were $\sim 5 \mu \mathrm{M}$ (Fig. 3A). Immunoblotting of the cell lysates indicated that GANT61 treatment led to PARP cleavage, suggesting that PC3 and DU145 cells underwent apoptosis (Fig. 3B). In addition, GANT61 treatment significantly decreased the efficiency of PC3 and DU145 cells to form liquid colonies $(\mathrm{P}<0.05$ or $\mathrm{P}<0.01$; Fig. $3 \mathrm{C}$ and D). These results indicate that the Gli inhibitor GANT61 may be used as a potential targeted therapy for androgen-independent prostate cancer.

mTOR/S6K1 signaling pathway is involved in the regulation of Glil expression in prostate cancer cells. Wang et al (8) reported that the activated $\mathrm{TNF} \alpha / \mathrm{mTOR} / \mathrm{S} 6 \mathrm{~K} 1$ signaling pathway promotes Gli1 transcriptional activity and oncogenic function in esophageal adenocarcinoma. To study the upstream pathway further, rather than the canonical $\mathrm{HH}$ pathway, involved in the regulation of Gli1 expression, it was investigated whether mTOR/S6K1 regulates Gli1 and its downstream target gene expression in prostate cancer cells. The documented Gli1 target genes include Glil and PTCH1, of which the corresponding proteins are important regulators of the canonical $\mathrm{HH}$ pathway itself. Other validated target genes include the cell cycle regulator cyclin D1 (CCND1), epithelial-mesenchymal transition regulator $S N A I L$, and self-renewal-associated molecules $N A N O G$ and $O C T 4$. PC3 cells were treated with $5 \mathrm{ng} / \mathrm{ml}$ TNF $\alpha$ for $24 \mathrm{~h}$ and it was identified that the PTCH1, CCNDI, $N-M Y C$ and Glil mRNA expression levels significantly increased, whereas the presence of rapamycin, an mTOR inhibitor, significantly decreased gene expression (Fig. 4A-D). Western blotting confirmed that $\mathrm{TNF} \alpha$ stimulation induced the activation of S6K1 and increased Gli1 protein expression, and that rapamycin inhibited $\mathrm{TNF} \alpha$-induced $\mathrm{S} 6 \mathrm{~K} 1$ phosphorylation and decreased Gli1 expression in PC3 cells (Fig. 4E). Similarly, depletion of S6K1 expression using siRNA, which was confirmed using RT-qPCR, markedly decreased 

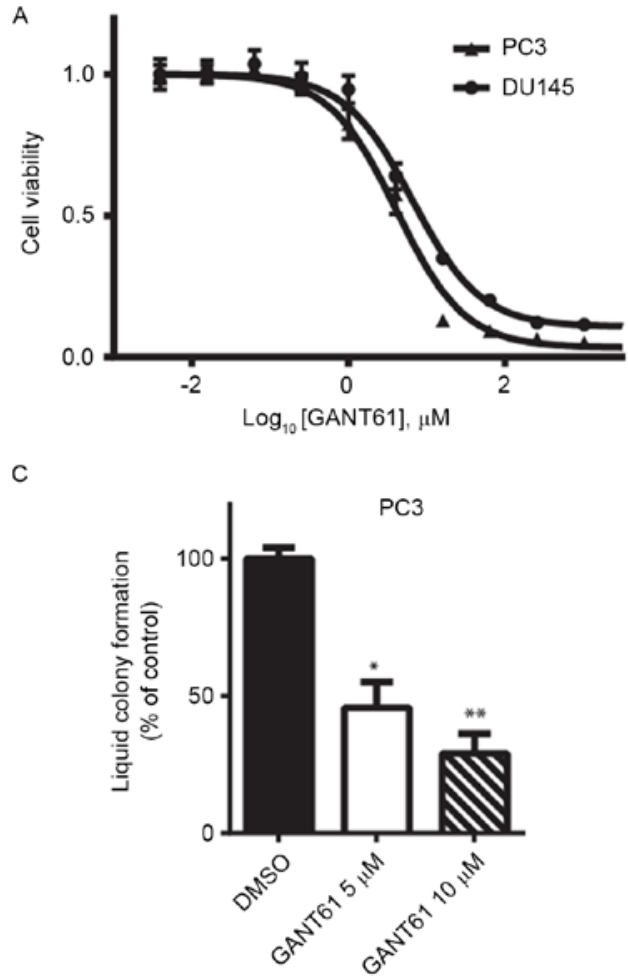

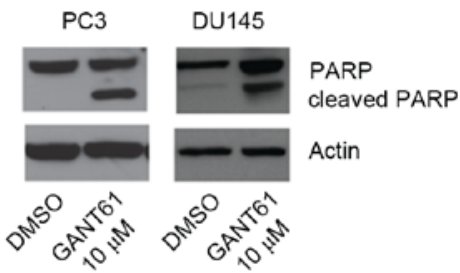

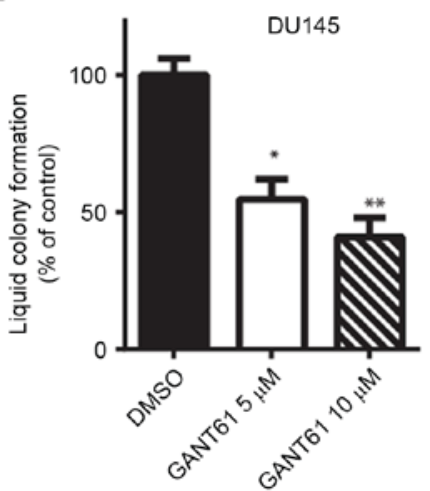

Figure 3. Gli inhibitor GANT61 decreases prostate cancer cell viability and colony formation in vitro. (A) PC3 and DU145 cells were seeded in 96-well plates and treated with the indicated concentration of GANT61 for 5 days. Cell viability was measured using CellTiter-Glo assays. (B) Immunoblotting for cleaved PARP revealed that GANT61 treatment for $48 \mathrm{~h}$ led to prostate cancer cell apoptosis compared with vehicle control. GANT61 significantly decreased (C) PC3 and (D) DU145 to form colonies in liquid medium. $\mathrm{P}<0.05,{ }^{\text {*" }} \mathrm{P}<0.01$ vs. control. PARP, poly(ADP-ribose) polymerase; DMSO, dimethyl sulfoxide.
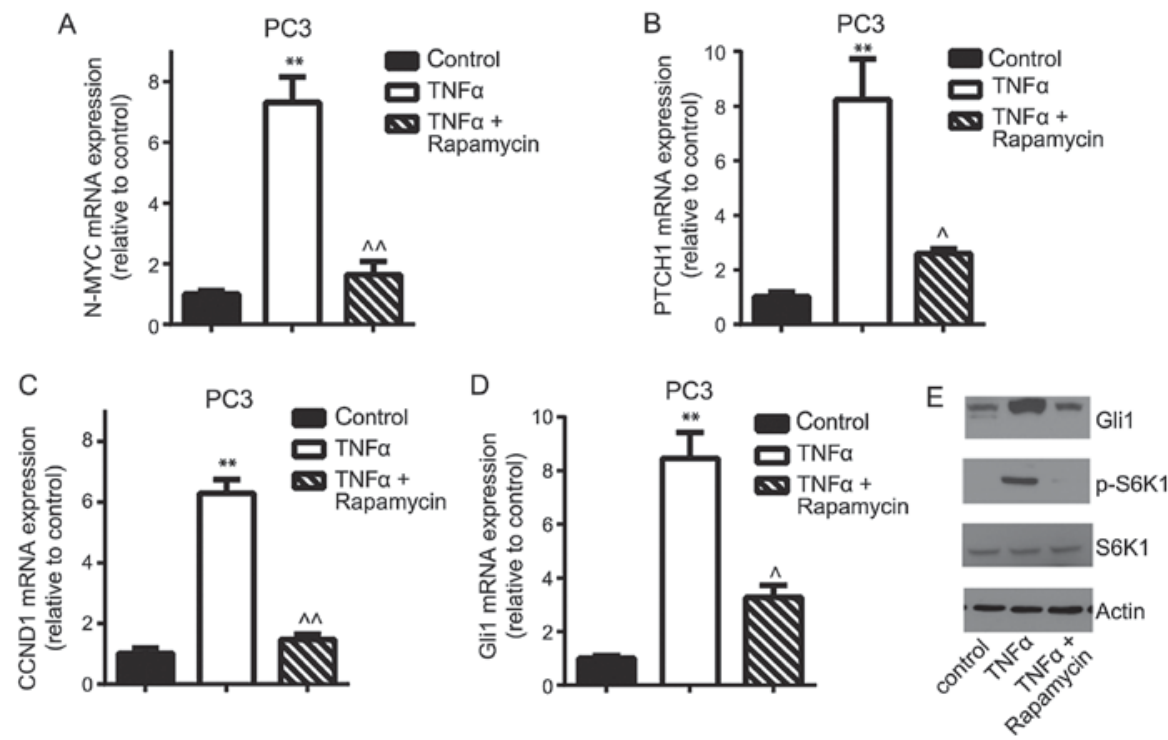

Figure 4. mTOR/S6K1 signaling pathway is involved in the regulation of Gli1 expression in PC3 cells. The reverse transcription-quantitative polymerase chain reaction demonstrated that $5 \mathrm{ng} / \mathrm{ml} \mathrm{TNF} \alpha$ treatment for $24 \mathrm{~h}$ stimulated the Gli1 downstream target genes and the Gli1 gene (A) $N$-MYC, (B) CCND1, (C) PTCH1 and (D) Glil mRNA expression compared with controls, whereas the mTOR inhibitor rapamycin decreased TNFa-induced mRNA expression. (E) Western blotting confirmed TNF $\alpha$-triggered phosphorylation of S6K1 and increased Gli1 expression. However, rapamycin inhibited TNFo-induced S6K1 activation and decreased Gli1 expression in $\mathrm{PC} 3$ cells. ${ }^{* *} \mathrm{P}<0.01$ vs. control; ${ }^{\wedge} \mathrm{P}<0.05,{ }^{\wedge} \mathrm{P}<0.01$ compared with $\mathrm{TNF} \alpha$ treatment. mTOR, mammalian target of rapamycin; S6K1, p70 ribosomal S6 kinase 1; Gli, glioma-associated oncogene; PTCH1, Patched 1; CCND1, cyclin D1; TNF $\alpha$, tumor necrosis factor $\alpha$; p-, phosphorylated.

TNF $\alpha$-induced Gli1 mRNA and protein expression (Fig. 5A and B). Consistent with genetic manipulation, pharmacological inhibition of mTOR/S6K1 pathway using the PI3K/mTOR dual inhibitor BEZ235 markedly decreased PC3 cell viability and
Gli1 protein expression (Fig. 5C and D). These results suggest that the TNF $\alpha / \mathrm{mTOR} / \mathrm{S} 6 \mathrm{~K} 1$ signaling pathway contributes to the regulation of Gli1 gene expression and its transcriptional activity in prostate cancer cells. 
A

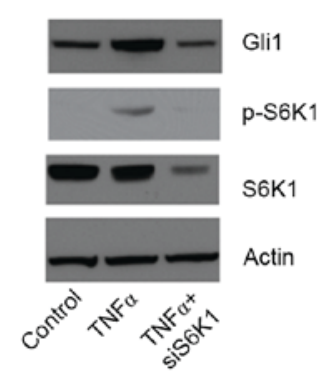

B

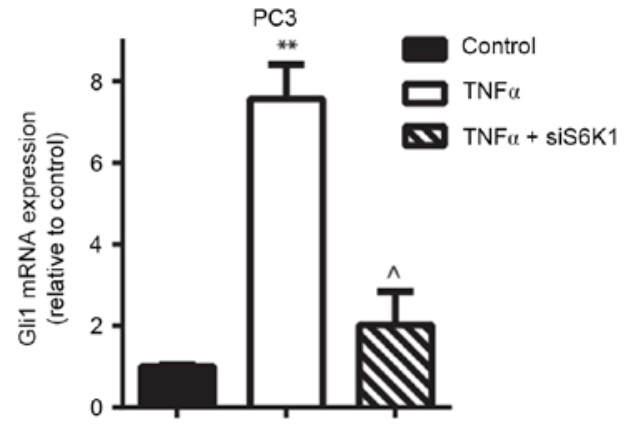

C

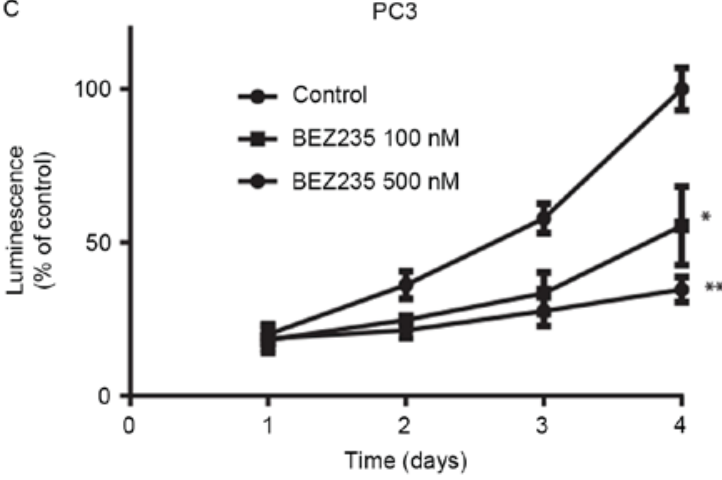

D Figure 5. Inhibiting the mTOR/S6K1 signaling pathway suppresses Gli1 expression and PC3 cell viability in vitro. (A) Reverse transcription-quantitative
polymerase chain reaction demonstrated that siS6K1 significantly decreased S6K1 mRNA expression in the presence of TNF $\alpha$. $\beta$-actin was used as a loading control. (B) Transient transfection of PC3 cells with siS6K1 significantly decreased TNF $\alpha$-induced Gli1 mRNA expression compared with scrambled siRNA control. (C) Similarly, the phosphoinositide 3-kinase/mTOR dual inhibitor BEZ235 treatment at the indicated concentrations suppressed PC3 cell viability. (D) BEZ235 decreased endogenous Gli1 protein expression in PC3 cells. $\beta$-actin was used as a loading control. ${ }^{*} \mathrm{P}<0.05$, ${ }^{* *} \mathrm{P}<0.01$ vs. control; ${ }^{\wedge} \mathrm{P}<0.05$ vs. $\mathrm{TNF} \alpha$ treatment. mTOR, mammalian target of rapamycin; S6K1, p70 ribosomal S6 kinase 1; Gli1, glioma-associated oncogene; TNF $\alpha$, tumor necrosis factor $\alpha$; siRNA, small interfering RNA; p-, phosphorylated; siS6K1, siRNA targeting S6K1.

\section{Discussion}

The results of the present study identified that Gli1 is overexpressed at the protein level in androgen-independent prostate cancer cell lines compared with androgen-dependent prostate cancer cells. Silencing of Gli1 inhibited prostate cancer cell proliferation and liquid colony formation in vitro. The Gli1/2-specific inhibitor GANT61 markedly decreased prostate cancer.cell viability by inducing cell apoptosis. Pharmacological and genetic manipulation of the TNFa/mTOR/S6K1 signaling pathway altered Gli1 expression and cancer cell survival. These results suggest that Gli1 is a suitable target for drug development for androgen-independent prostate cancer.

Previous studies have indicated that the $\mathrm{HH}$ pathway contributes to the initiation as well as the progression of prostate cancer (17-19). Previous studies have assessed the protein expression of the $\mathrm{HH}$ components in tissue microarrays and identified that the expression of SHH, SMO and PTCH1 in the tumor was upregulated compared with adjacent normal tissue. However, stromal PTCH1, SMO and Gli1 expression were downregulated in the tumor compared with normal tissue $(20,21)$. Canonical $\mathrm{HH}$ pathway activation appears to be more marked in late-stage prostate cancer $(22,23)$. In addition, results of the present study and previous studies observed a potential association between $\mathrm{HH}$ signaling component expression and androgen-independent prostate cancer cells $(1,15)$. Long-term androgen deprivation may induce an upregulation of $\mathrm{HH}$ signaling in human specimens and in cell lines. Inhibition of HH signaling led to a decrease in androgen receptor activation, partly because of the interaction between Gli1/2 and the androgen receptor (24). The present study provides experimental evidence for the application of Gli1/2 inhibitors in the treatment of androgen-insensitive prostate cancer.

In the present study, the main focus was on Gli1 activity and function in androgen-independent prostate cancer lines and it was identified that the Gli1/2-specific small-molecule inhibitor GANT61 significantly impaired prostate cancer cell proliferation. Further studies are required to elucidate the function of Gli1 and pharmacological effect of GANT61 in vivo. Gli2 has been demonstrated to be involved in the malignant transformation of prostate cancer cells. Thiyagarajan et al (25) reported that knockdown of Gli2 in prostate cancer cells suppressed tumor growth in vitro and in vivo. The mechanisms by which the $\mathrm{HH}$ signaling pathway serves a role in the initiation and progression of prostate cancer include the anti-apoptotic effect and the inhibition of invasiveness and metastasis. Karlou et al (26) also demonstrated that the SMO inhibitor GDC-0449 exhibited the ability to inhibit prostate cancer xenograft tumor growth. Despite the promising results of $\mathrm{HH}$ inhibition in prostate cancer cell lines and mouse models, this has not been fully translated into the clinic.

Previously, aberrant $\mathrm{HH}$ signaling in prostate tumors was reported to be ligand-dependent, although it is controversial whether this is in a paracrine or autocrine manner (3). In fact, the $\mathrm{HH}$ pathway is part of a complex signaling network that remains incompletely understood (9). The PI3K/Akt and 
Ras/MAPK/ERK kinase (MEK)/ERK signaling pathways have been demonstrated to activate Gli1 in a SMO-independent manner $(10,11)$. In prostate cancer, alterations in the PI3K/Akt signaling pathway are common in primary and metastatic lesions (27). The Ras/MEK/ERK pathway is also constitutively activated in a number of prostate tumor tissues and appears to exhibit an association with advanced and androgen-independent prostate cancer (27). Furthermore, the tumor suppressor PTEN, which is a negative regulator of the signaling pathway, is lost in $<80 \%$ of prostate cancers, leading to constitutive activation of Akt pathway (22); however, the underlying molecular mechanism is not fully understood. In the present study, it was identified that the TNF $\alpha / \mathrm{mTOR} / \mathrm{S} 6 \mathrm{~K} 1$ signaling pathway was partly responsible for the aberrant overexpression of Gli protein. Inhibiting mTOR/S6K1 activity markedly blocked Gli1 function in androgen-independent prostate cancer cells. The next step is to determine the in vivo effect of inhibiting the mTOR/S6K1 signaling pathway on prostate xenograft tumor growth. Additionally, Narita et al (28) have demonstrated that inhibition of HH signaling may increase the chemosensitivity of prostate cancer cells.

The results of the present study indicate that Gli1 transcriptional activity is critical in androgen-independent prostate cancer cell proliferation. The PI3K/mTOR/S6K1 signaling pathway is implicated in the regulation of Glil expression and functions in a SMO-independent manner. Blocking the non-canonical HH pathway or directly inhibiting Gli1 transcriptional activity may open a new avenue for targeted therapies. Further study using mouse models may lead to a better understanding of the role of Gli1 in prostate cancer growth and eventually to inhibitors that may be used as tools for research or treatment.

\section{Acknowledgements}

This research was supported by grants from the National Natural Science Foundation of China (grant no. 81201767) and the Joint Special Funds for the Department of Science and Technology of Yunnan Province-Kunming Medical University (grant nos. 2011FB202, 2013FZ277 and 2017FE467-191).

\section{References}

1. Gonnissen A, Isebaert S and Haustermans K: Hedgehog signaling in prostate cancer and its therapeutic implication. Int J Mol Sci 14: 13979-14007, 2013.

2. Guo R, Cai L, Fan Y, Jin J, Zhou L and Zhang K: Magnetic resonance imaging on disease reclassification among active surveillance candidates with low-risk prostate cancer: A diagnostic meta-analysis. Prostate Cancer Prostatic Dis 18: 221-228, 2015.

3. Chen M, Carkner R and Buttyan R: The hedgehog/Gli signaling paradigm in prostate cancer. Exp Rev Endocrinol Metab 6 : 453-467, 2011.

4. Kim TJ, Lee JY, Hwang TK, Kang CS and Choi YJ: Hedgehog signaling protein expression and its association with prognostic parameters in prostate cancer: A retrospective study from the view point of new 2010 anatomic stage/prognostic groups. J Sur Oncol 104: 472-479, 2011.

5. Jiang J and Hui CC: Hedgehog signaling in development and cancer. Dev Cell 15: 801-812, 2008.

6. Onishi $\mathrm{H}$ and Katano M: Hedgehog signaling pathway as a therapeutic target in various types of cancer. Cancer Sci 102: 1756-1760, 2011

7. Rubin LL and de Sauvage FJ: Targeting the Hedgehog pathway in cancer. Nat Rev Drug Discov 5: 1026-1033, 2006.
8. Wang Y, Ding Q, Yen CJ, Xia W, Izzo JG, Lang JY, Li CW, Hsu JL, Miller SA, Wang X, et al: The crosstalk of mTOR/S6K1 and Hedgehog pathways. Cancer Cell 21: 374-387, 2012.

9. Lauth M and Toftgård R: Non-canonical activation of GLI transcription factors: Implications for targeted anti-cancer therapy. Cell 6: 2458-2463, 2007.

10. Riobo NA, Haines GM and Emerson CP Jr: Protein kinase C-delta and mitogen-activated protein/extracellular signal-regulated kinase-1 control GLI activation in hedgehog signaling. Cancer Res 66: 839-845, 2006.

11. Riobo NA, Lu K, Ai X, Haines GM and Emerson CP Jr: Phosphoinositide 3-kinase and Akt are essential for Sonic Hedgehog signaling. Proc Natl Acad Sci USA 103: 4505-4510, 2006.

12. Yang L, Xie G, Fan Q and Xie J: Activation of the hedgehog-signaling pathway in human cancer and the clinical implications. Oncogene 29: 469-481, 2010.

13. Vandesompele J, De Preter K, Pattyn F, Poppe B, Van Roy N, De Paepe A and Speleman F: Accurate normalization of real-time quantitative RT-PCR data by geometric averaging of multiple internal control genes. Genome Biol 3: RESEARCH0034, 2002.

14. Ebrahimi A, Larijani L, Moradi A and Ebrahimi MR: Hedgehog signalling pathway: Carcinogenesis and targeted therapy. Iran J Cancer Prev 6: 36-43, 2013.

15. Chen G, Goto Y, Sakamoto R, Tanaka K, Matsubara E, Nakamura M, Zheng H, Lu J, Takayanagi R and Nomura M: GLI1, a crucial mediator of sonic hedgehog signaling in prostate cancer, functions as a negative modulator for androgen receptor. Biochem Biophys Res Commun 404: 809-815, 2011.

16. Lauth M, Bergström A, Shimokawa T and Toftgård R: Inhibition of GLI-mediated transcription and tumor cell growth by small-molecule antagonists. Proc Natl Acad Sci USA 104: 8455-8460, 2007.

17. Thayer SP, di Magliano MP, Heiser PW, Nielsen CM, Roberts DJ, Lauwers GY, Qi YP, Gysin S, Fernández-del Castillo C, Yajnik V, et al: Hedgehog is an early and late mediator of pancreatic cancer tumorigenesis. Nature 425: 851-856, 2003.

18. Pasca di Magliano M and Hebrok M: Hedgehog signalling in cancer formation and maintenance. Nat Rev Cancer 3: 903-911, 2003.

19. Podlasek CA, Barnett DH, Clemens JQ, Bak PM and Bushman W: Prostate development requires Sonic hedgehog expressed by the urogenital sinus epithelium. Dev Biol 209: 28-39, 1999.

20. Datta S and Datta MW: Sonic Hedgehog signaling in advanced prostate cancer. Cell Mol Life Sci 63: 435-448, 2006.

21. Sheng T, Li C, Zhang X, Chi S, He N, Chen K, McCormick F, Gatalica Z and Xie J: Activation of the hedgehog pathway in advanced prostate cancer. Mol Cancer 3: 29, 2004.

22. Karhadkar SS, Bova GS, Abdallah N, Dhara S, Gardner D, Maitra A, Isaacs JT, Berman DM and Beachy PA: Hedgehog signalling in prostate regeneration, neoplasia and metastasis. Nature 431: 707-712, 2004.

23. Fan L, Pepicelli CV, Dibble CC, Catbagan W, Zarycki JL, Laciak R, Gipp J, Shaw A, Lamm ML, Munoz A, et al: Hedgehog signaling promotes prostate xenograft tumor growth. Endocrinology 145: 3961-3970, 2004.

24. Efstathiou E, Karlou M, Wen S, Hoang A, Pettaway CA Pisters LL, Maity S, Troncoso P and Logothetis CJ: Integrated Hedgehog signaling is induced following castration in human and murine prostate cancers. Prostate 73: 153-161, 2013.

25. Thiyagarajan S, Bhatia N, Reagan-Shaw S, Cozma D, Thomas-Tikhonenko A, Ahmad N and Spiegelman VS: Role of GLI2 transcription factor in growth and tumorigenicity of prostate cells. Cancer Res 67: 10642-10646, 2007.

26. Karlou M, Lu JF, Wu G, Maity S, Tzelepi V, Navone NM, Hoang A, Logothetis CJ and Efstathiou E: Hedgehog signaling inhibition by the small molecule smoothened inhibitor GDC-0449 in the bone forming prostate cancer xenograft MDA PCa 118b. Prostate 72: 1638-1647, 2012.

27. Taylor BS, Schultz N, Hieronymus H, Gopalan A, Xiao Y, Carver BS, Arora VK, Kaushik P, Cerami E, Reva B, et al: Integrative genomic profiling of human prostate cancer. Cancer Cell 18: 11-22, 2010.

28. Narita S, So A, Ettinger S, Hayashi N, Muramaki M, Fazli L, Kim Y and Gleave ME: GLI2 knockdown using an antisense oligonucleotide induces apoptosis and chemosensitizes cells to paclitaxel in androgen-independent prostate cancer. Clin Cancer Res 14: 5769-5777, 2008. 